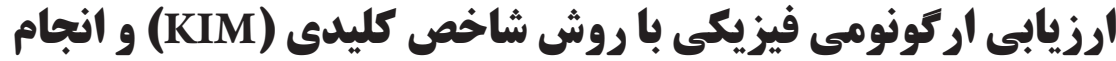

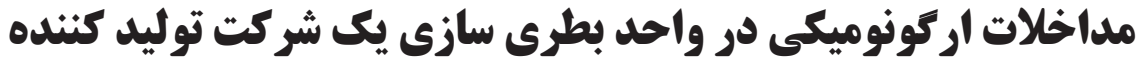

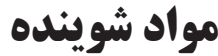

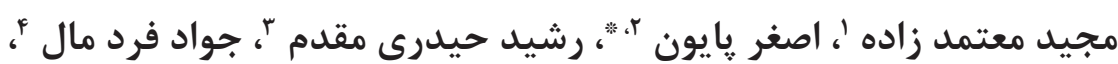

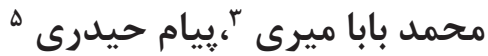

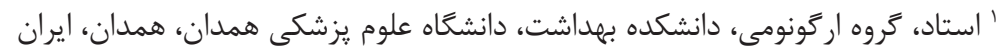

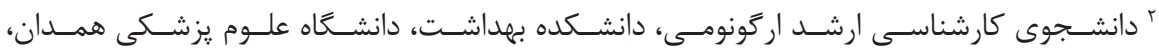

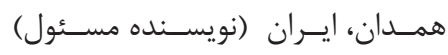

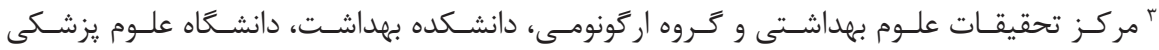

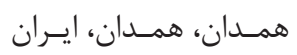

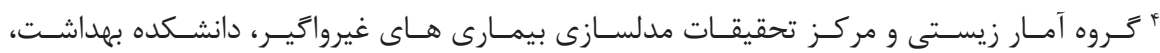

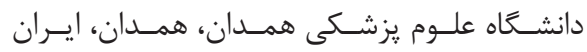

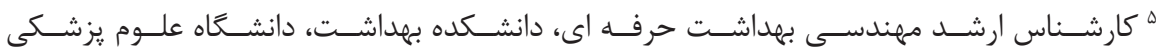

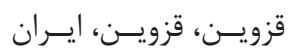

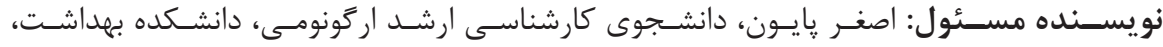

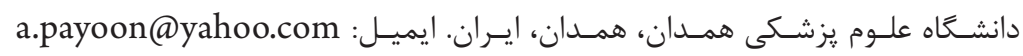

DOI: $10.21859 /$ joe- 05016

$$
\text { جكيده }
$$

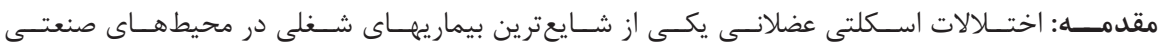

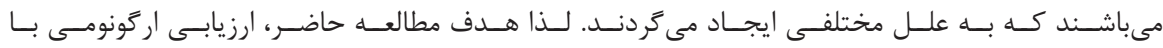

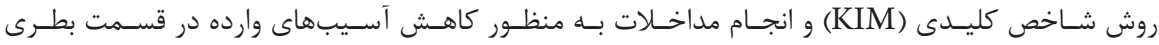

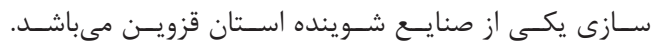

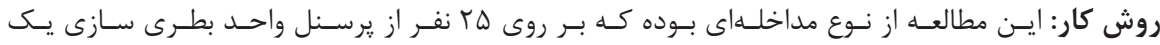

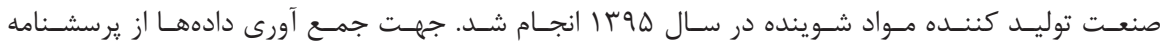

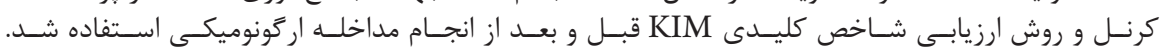

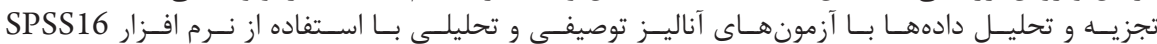

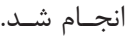

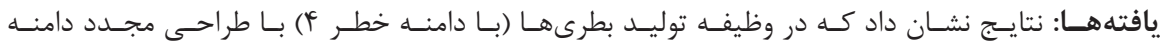

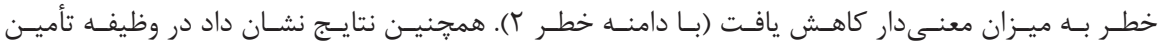

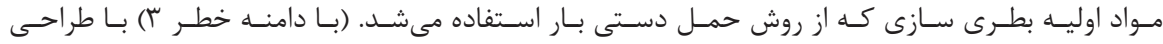

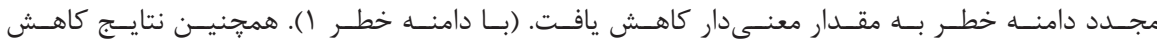

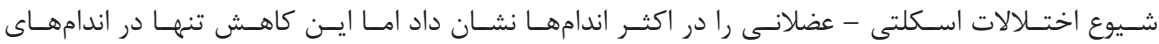

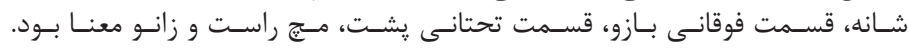

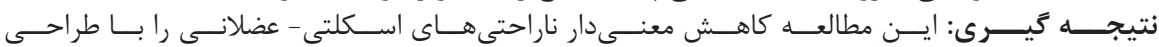

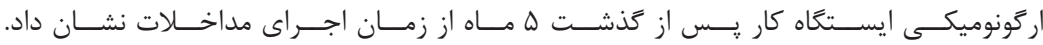

تاريخ دريافت مقاله: |||||||هوس||

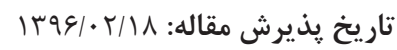

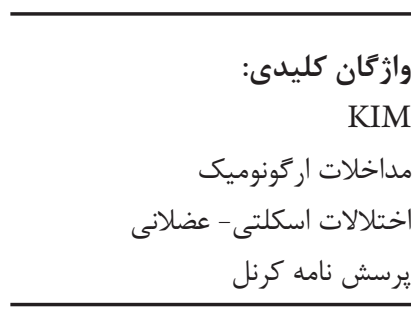

تممدانى ححفوظ نشر براى دانشكاه علوم يزشكى

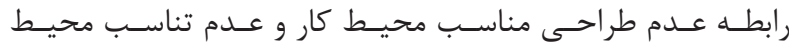

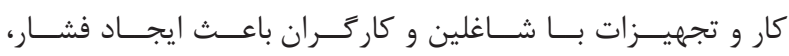

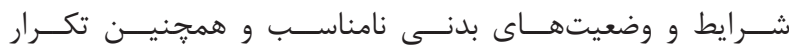

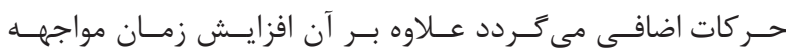

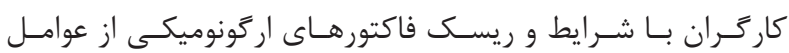

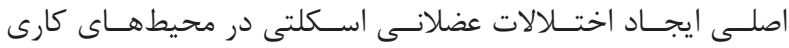

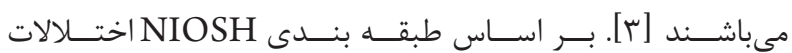

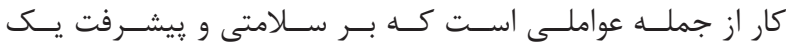

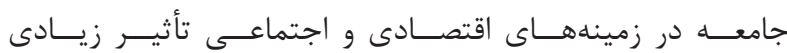

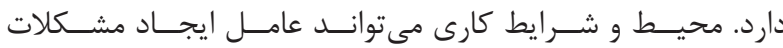

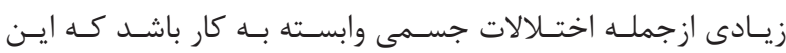

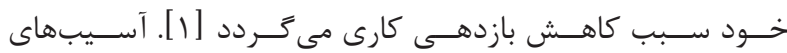

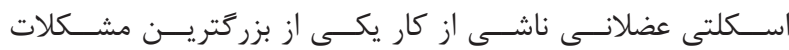

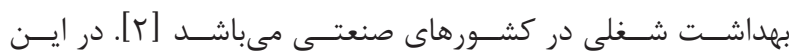




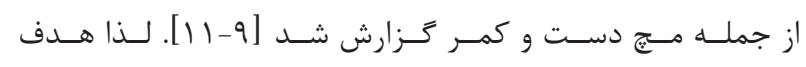

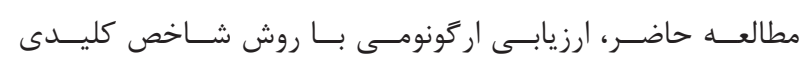

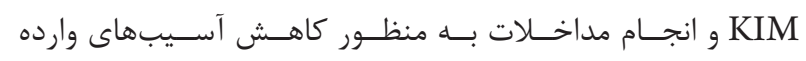

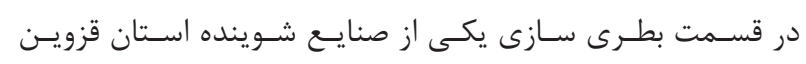
مى باشــد.

\section{روش كار}

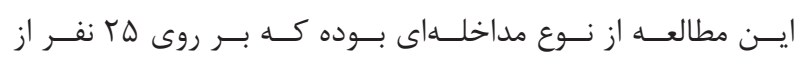

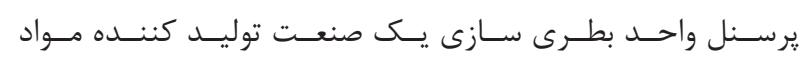

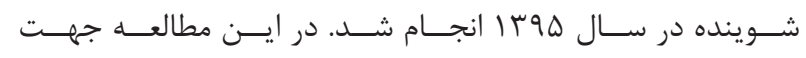

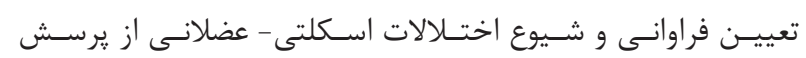

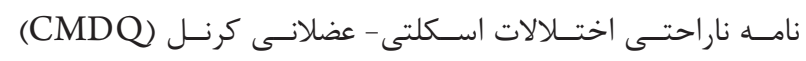

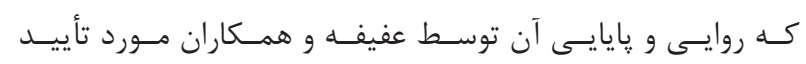

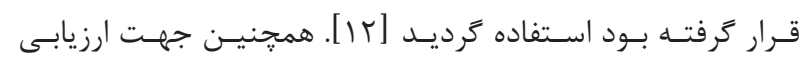

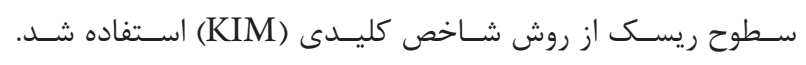

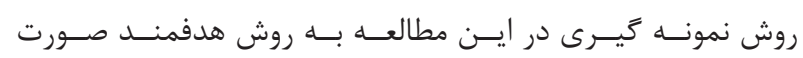

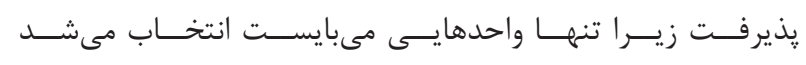

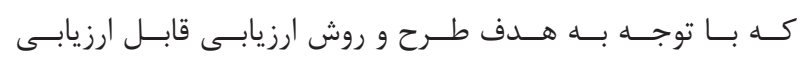

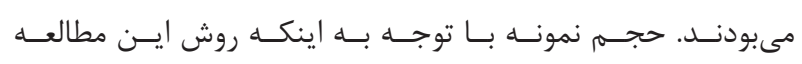

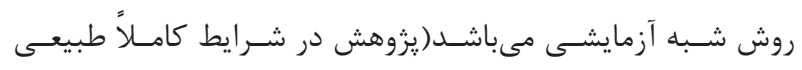

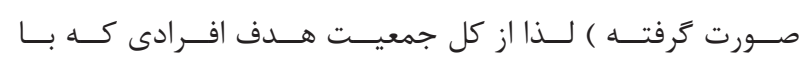

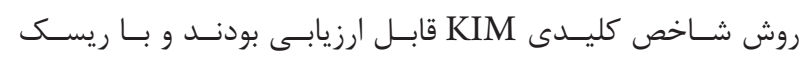

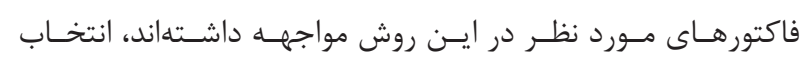

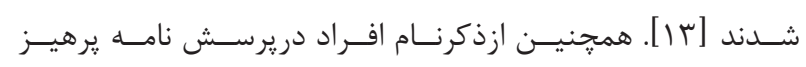

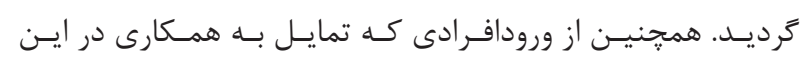

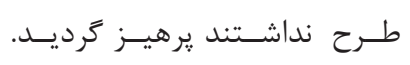

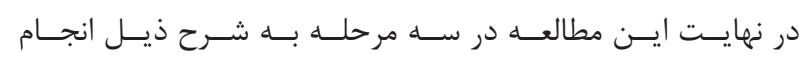
كرديسد:

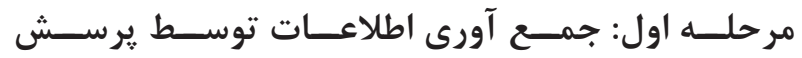

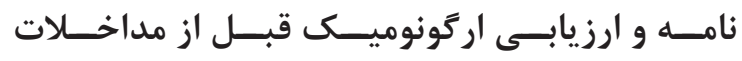

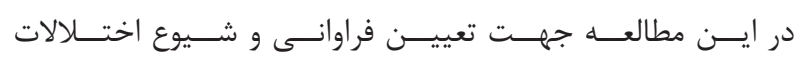

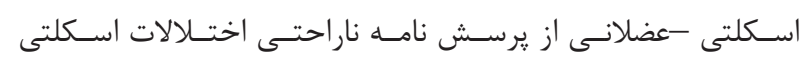

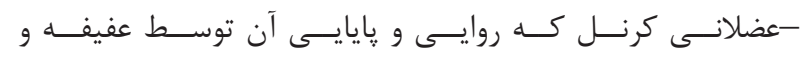

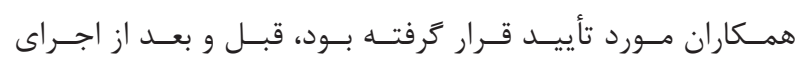

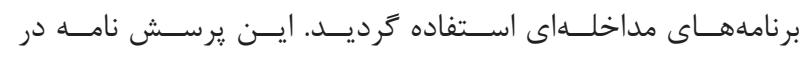

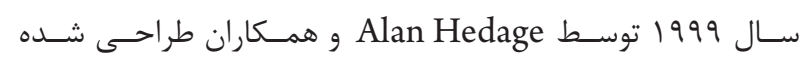

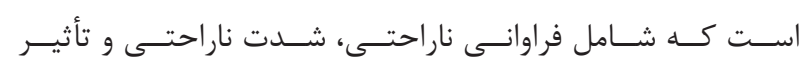

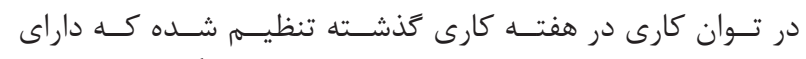

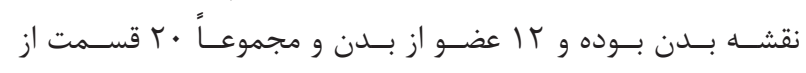

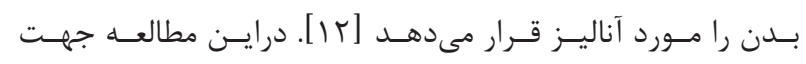

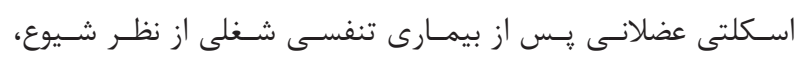

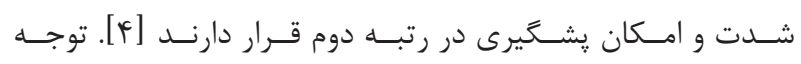

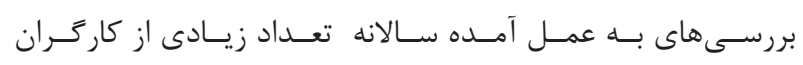

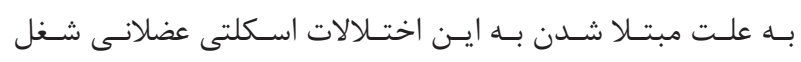

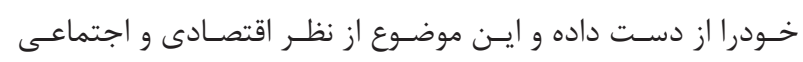

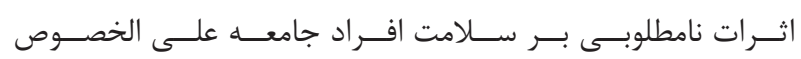

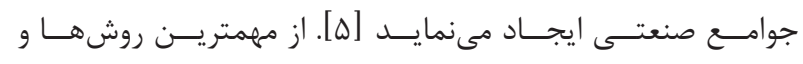

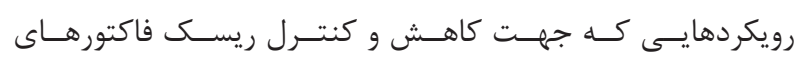

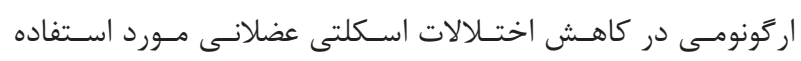

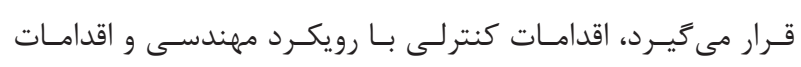

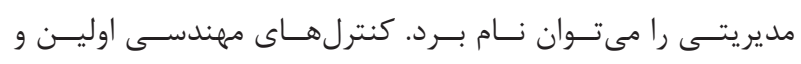

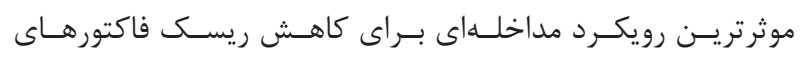

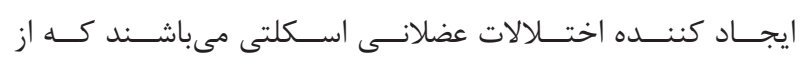

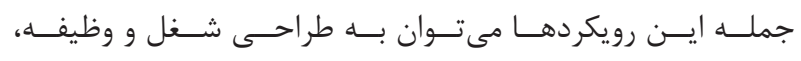

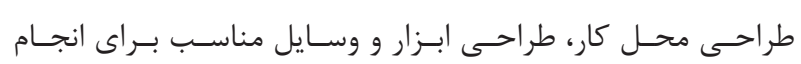

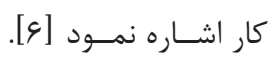

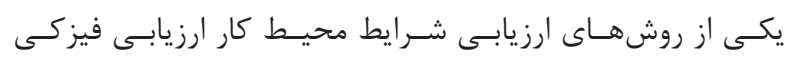

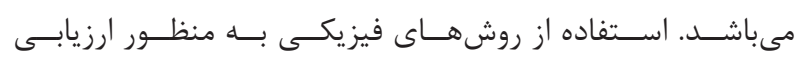

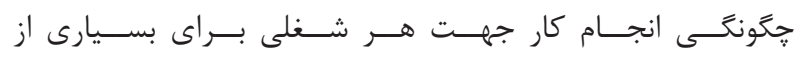

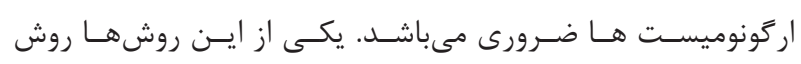

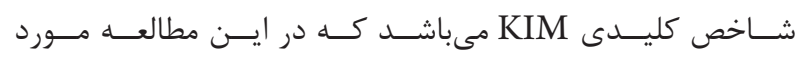

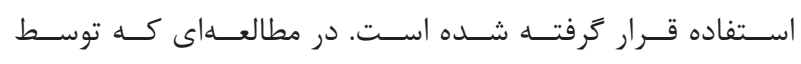

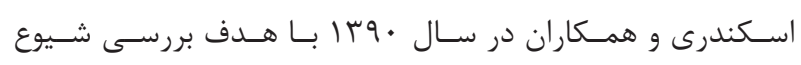

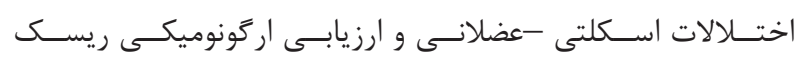

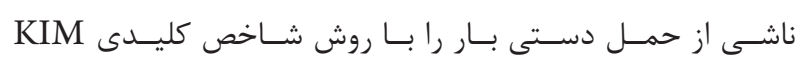

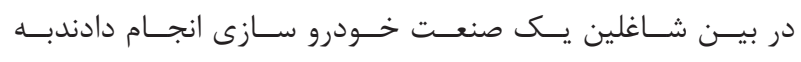

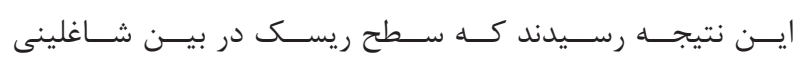

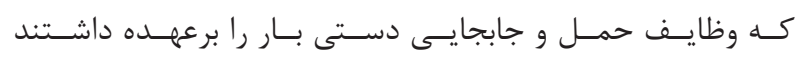

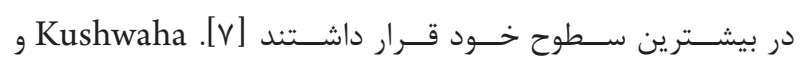

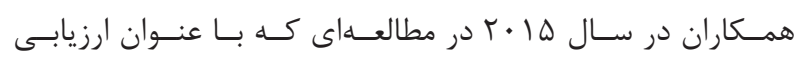

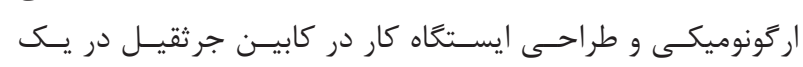

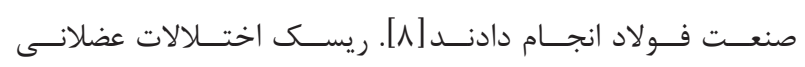

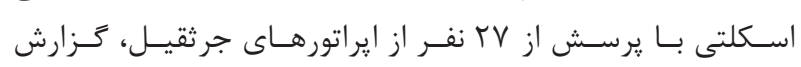

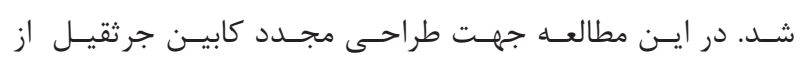

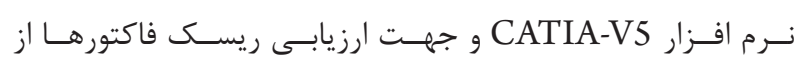

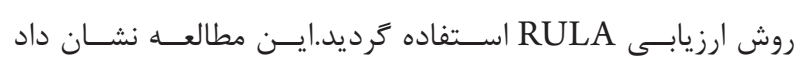

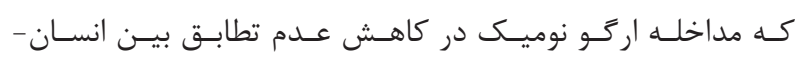

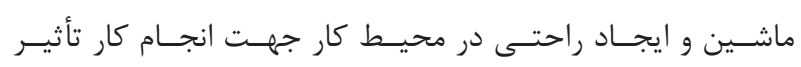

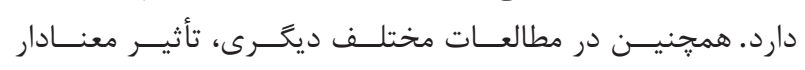

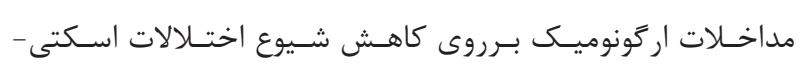

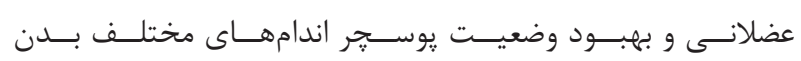




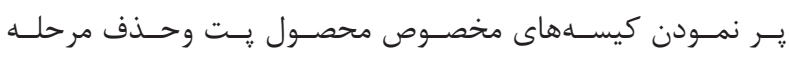

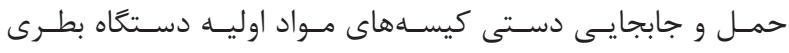

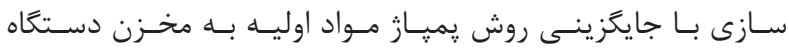

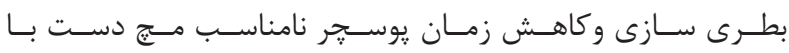

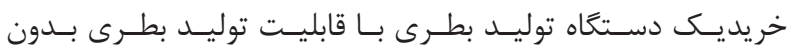

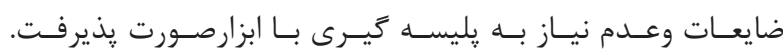
مر حلــه ســوم: جمــع آورى اطلاعـات و ارزيابــى تأثيــــ مد ا خلات مرحله

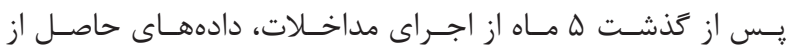

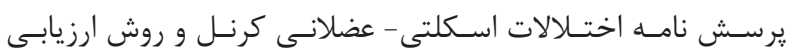

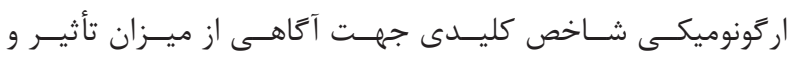

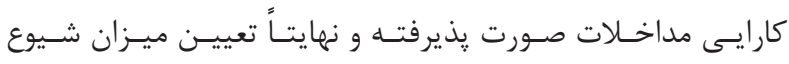

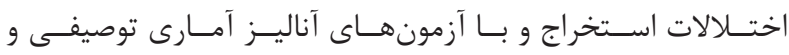

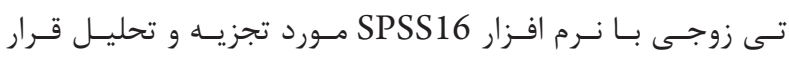
كرفتنـد.

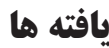

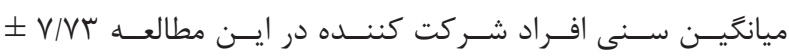

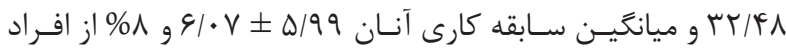

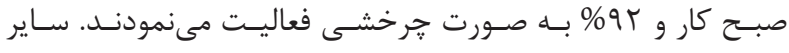

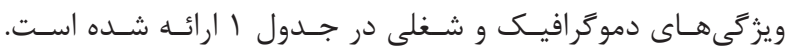

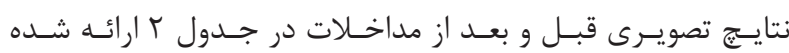

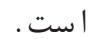

جدول ا: ويزَكى هاى دموكرافيك و شغلى افراد مورد مطالعه (D = (n)

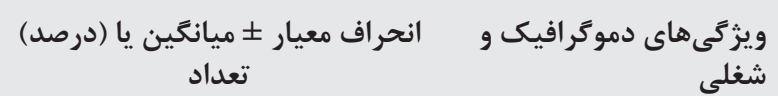
$\mid r / \cdots \pm \cdot \%$ زمان كار

TT/FA $\pm V / V T$ سن $9 / \cdot V \pm 0 / 99$

سابقه كار

\begin{tabular}{|c|c|}
\hline & شيفت كار \\
\hline$r(\Lambda)$ & روز كار \\
\hline$\cdot(\cdot)$ & عصر كار \\
\hline$\cdot(\cdot)$ & شب كار \\
\hline \multirow[t]{2}{*}{ Tr(qT) } & קرخشى \\
\hline & تحصيلات \\
\hline$r(\mid r)$ & ابتدايى \\
\hline $19\left(9 \varphi^{p}\right)$ & متوسطه \\
\hline$G\left(Y F^{\prime}\right)$ & دانشعاهى \\
\hline
\end{tabular}

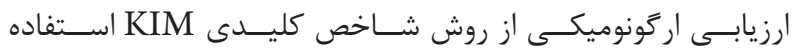

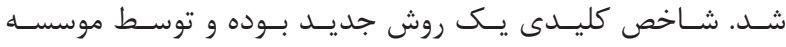

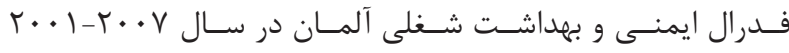

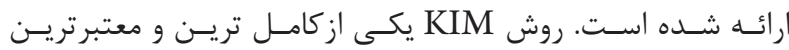

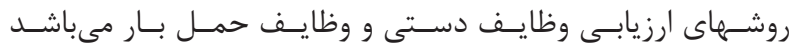

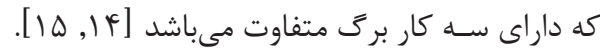

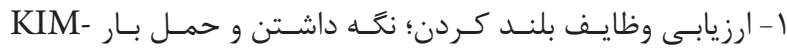
LHC K- ارزيابى وظايف كشـيدن / هل دادن بار KIM-PP

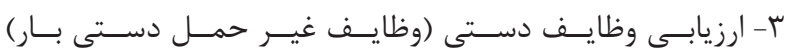
KIM-MHO

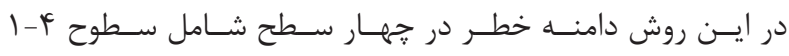

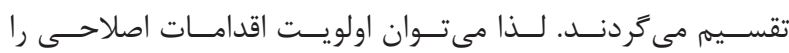

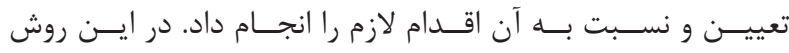

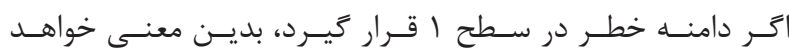

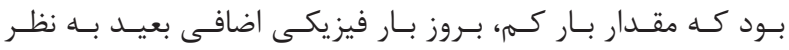

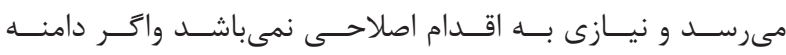

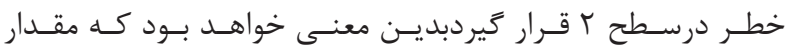

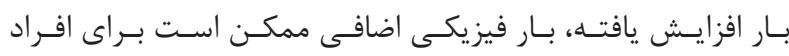

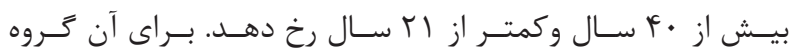

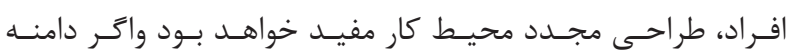

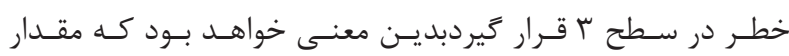

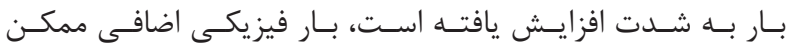

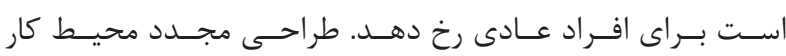

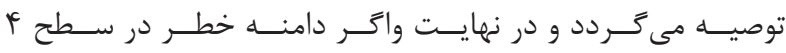

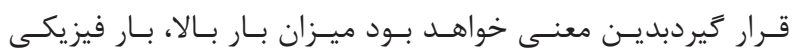

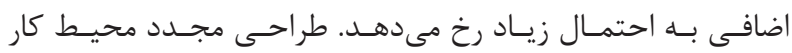

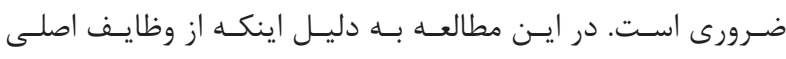

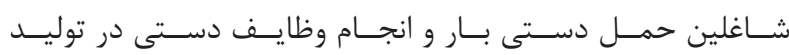

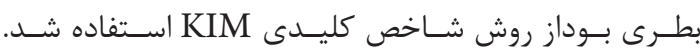
مر حله دوم: اجراى مداخلات ارَّونوميك

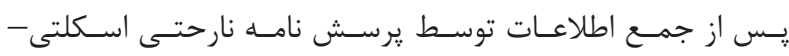

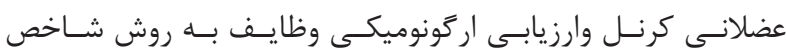

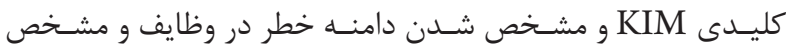

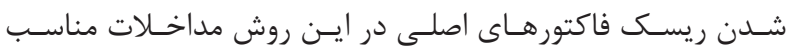

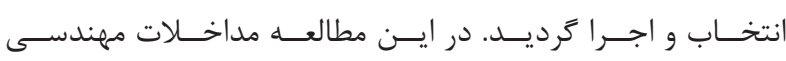

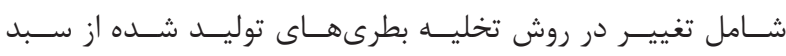

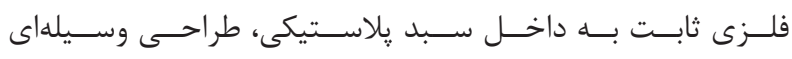

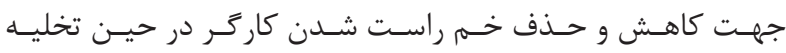

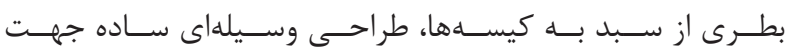

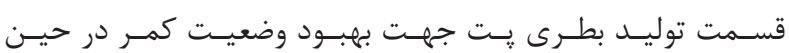




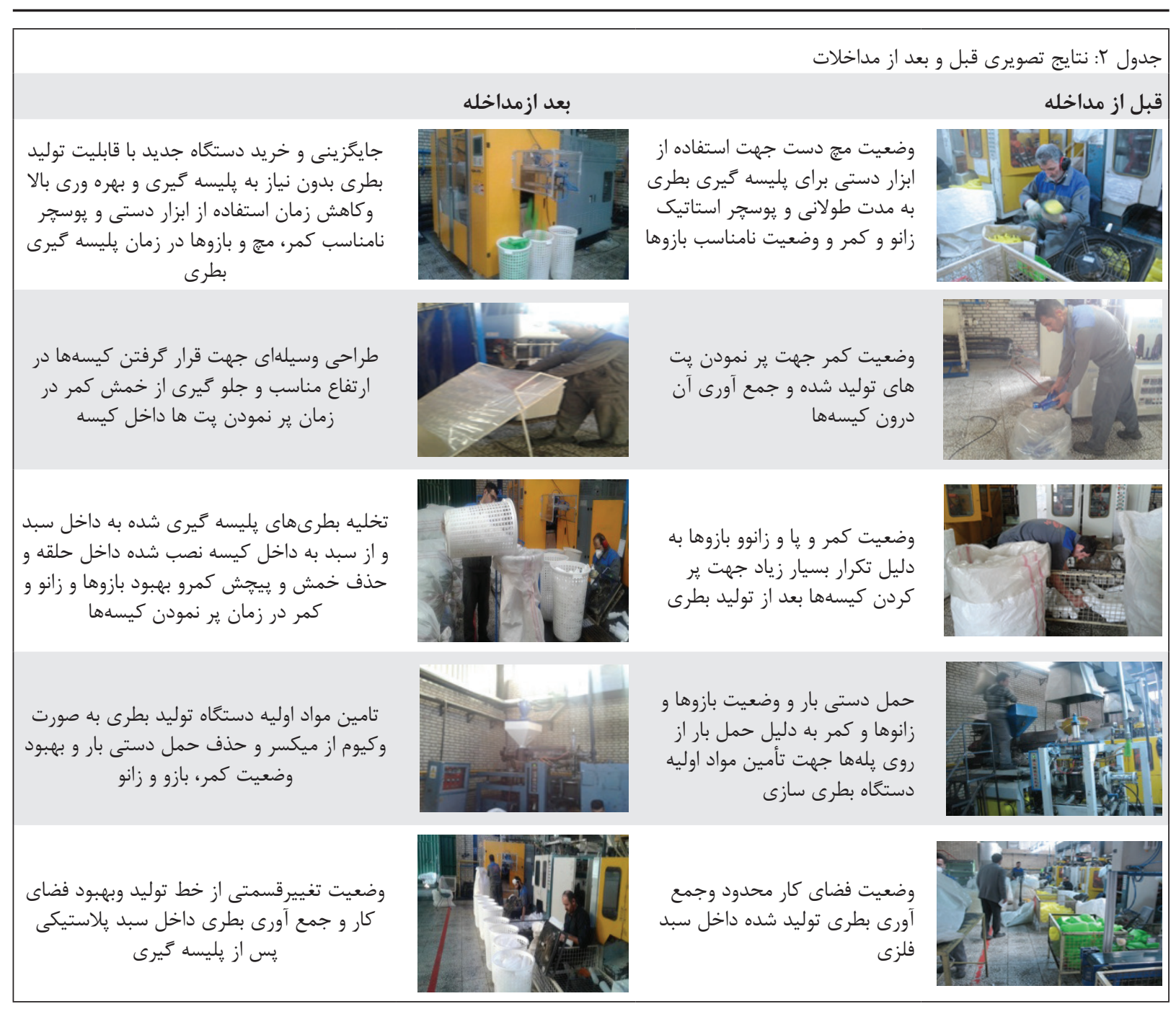

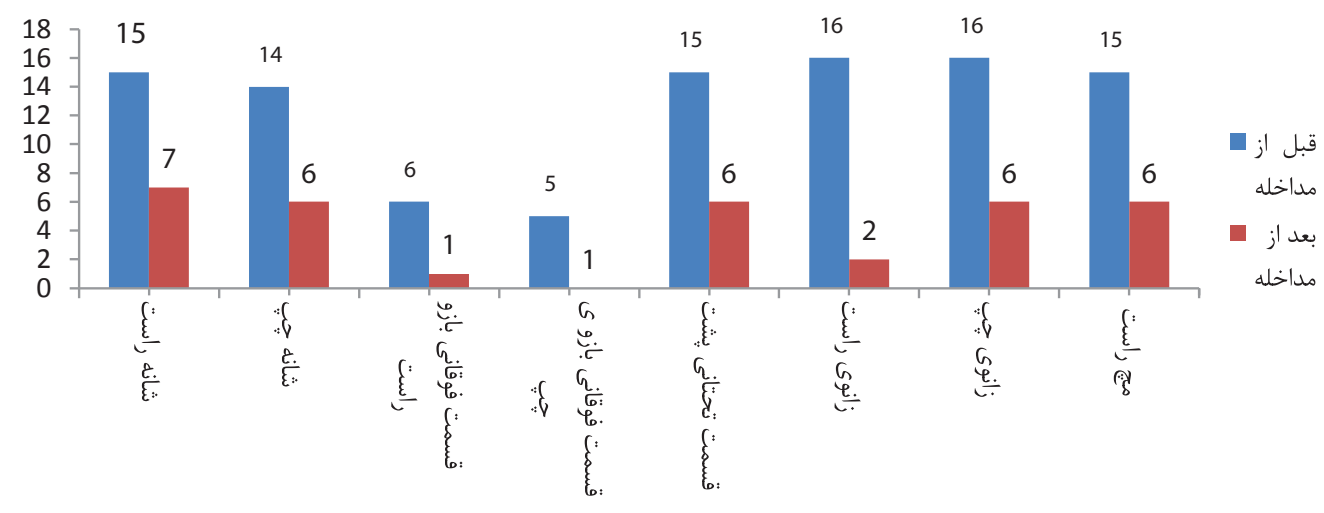

تصوير ا: مقايسه تعداد افراد داراى ناراحتىهاى اسكلتى-عضلانى در قبل و بعد از اجراى مداخلات

روش شـاخص كليـدى در قبـل و بعـد ازاجـــاى مداخـلات در جدول

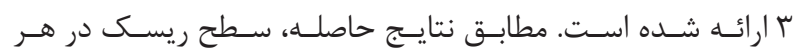
دو وظيفـه اصلى كاهـش نشـان داد.
تأثيـر مداخـلات بـر روى شـيوع اختـلالات اندامهــاى مختلـف بــن

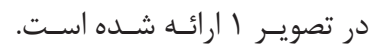

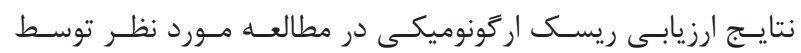




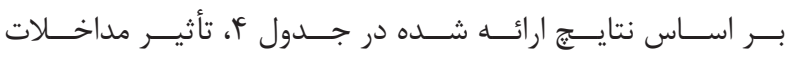

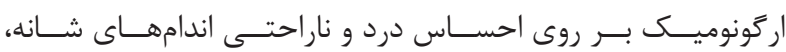

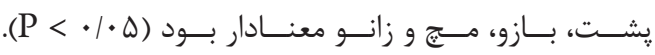

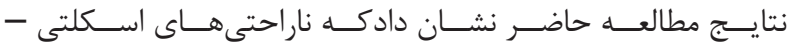

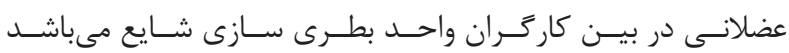

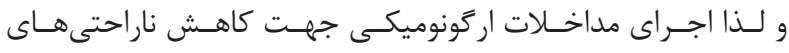

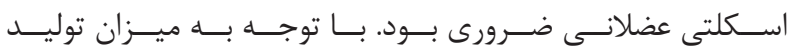

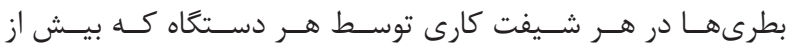

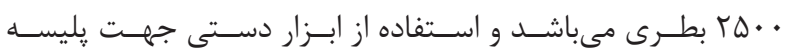

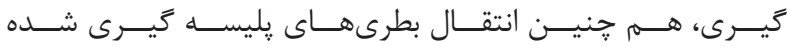

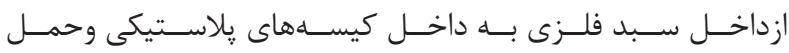

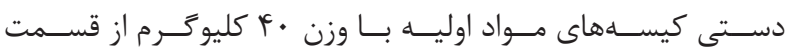

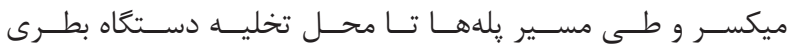

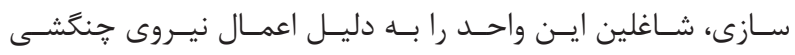

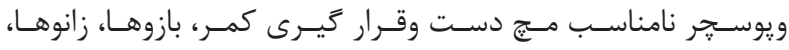

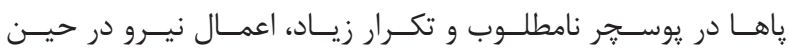

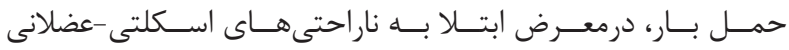

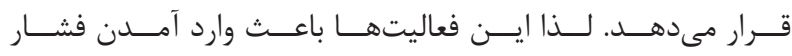

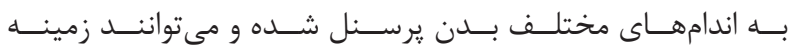

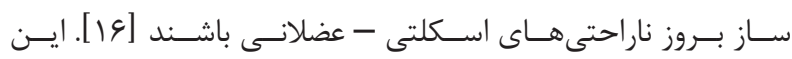

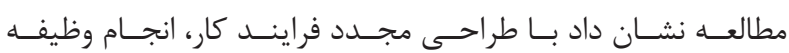

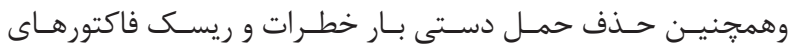

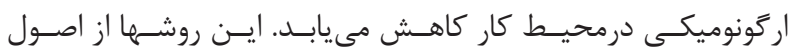

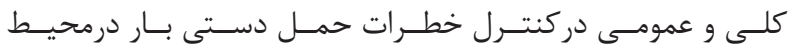

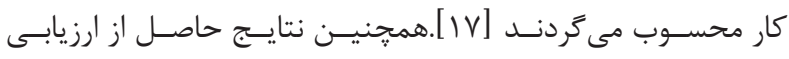

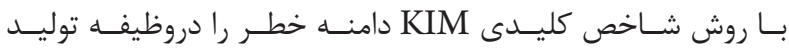

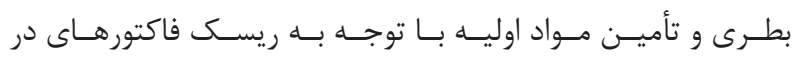

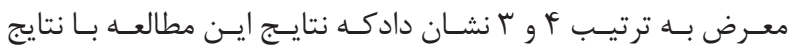

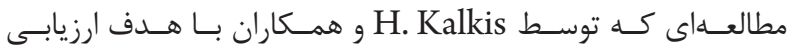

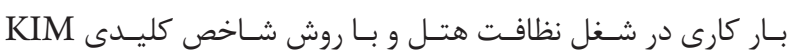

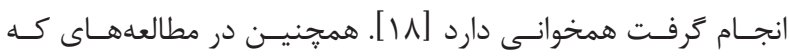

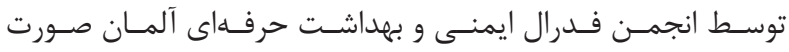

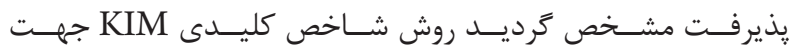

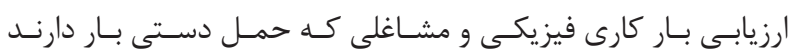

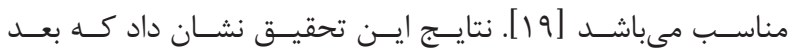

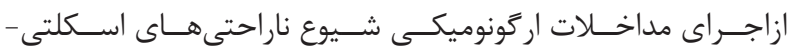

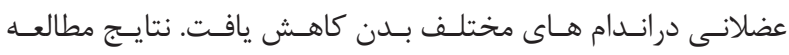

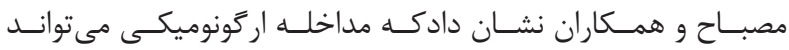

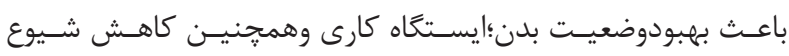

\begin{tabular}{|c|c|c|c|}
\hline كليدى و & 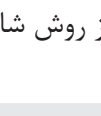 & 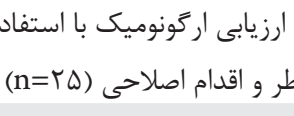 & جدول سا: نتاي \\
\hline سجر & امتياز & وظيفه/نوع فعاليت & 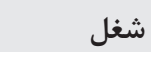 \\
\hline 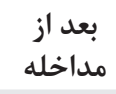 & 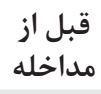 & & \\
\hline r & r & وظيفه توليد بطرى و جمع آن & بطرى سازى \\
\hline 1 & r & وظيفَه تأمين مواد اوليه & بطرى سازى \\
\hline
\end{tabular}

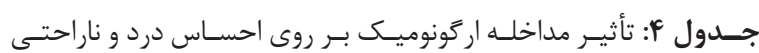

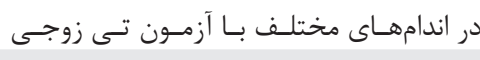

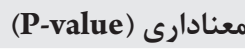

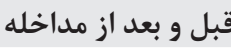

$\cdot / r \pi \Delta$ كردن شانه

$\cdot / \cdot T$ راست

. ج

$.1 .9 \mathrm{~V}$

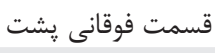

قسمت فوقانى بازو

. راست

.$/ 4 T$ ج

$\cdot 1 \cdot \cdot 1$ قسمت تحتانى پشت

.$/ 4 \Lambda$. ساعد

.1 .41 راست ק

. H TY راست

$\cdot \pi$. ج ק

- /ATF باسن

- rTAS راست

. IFIV च زانو

.$/ .4 F$ راست

.$/ r \mu$ ק

- IFT قسمت تحتانى ها

.1 .99 راست च

./IV.

./IV. ج 
حــف ترديـــ [عץ]. ايـن مطالعـه نشـان داد بــا تغييـر سـاده در

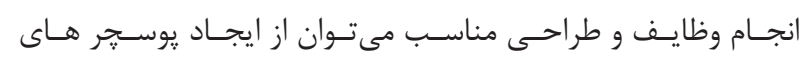

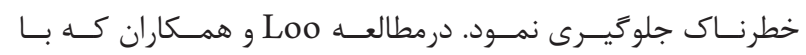

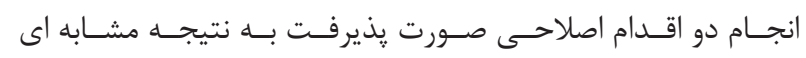

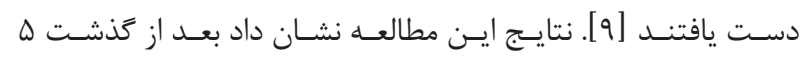

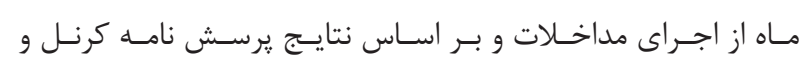

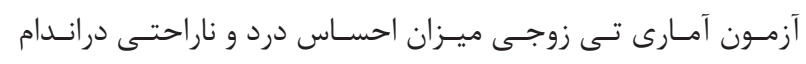

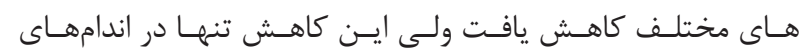

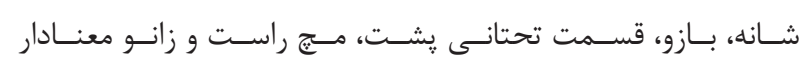

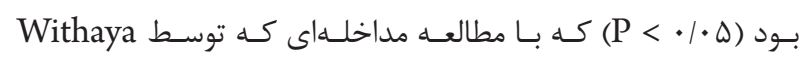
Chanchia

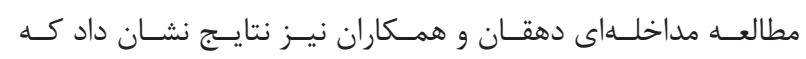

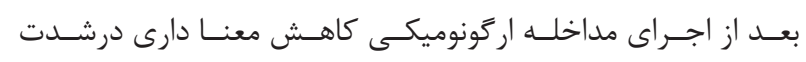

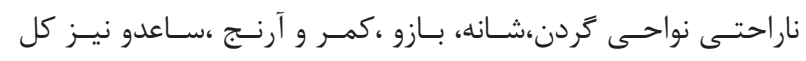

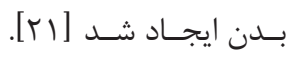

\section{نتيجه تيرى}

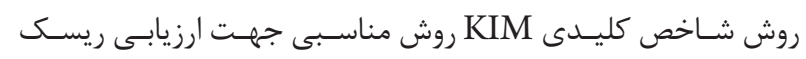

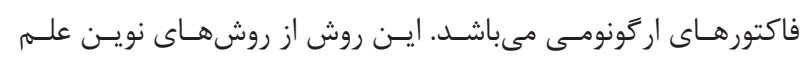

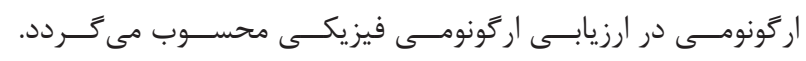

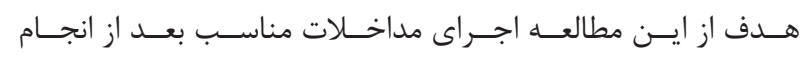

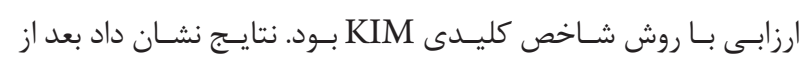

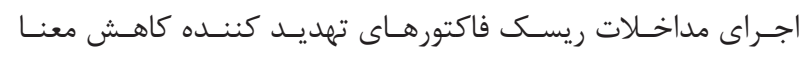

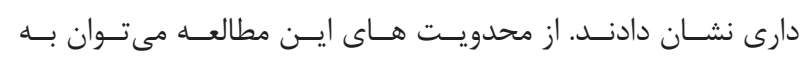

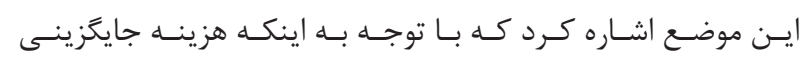

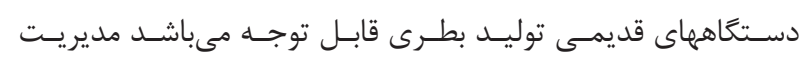

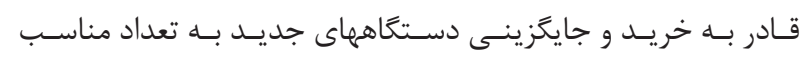

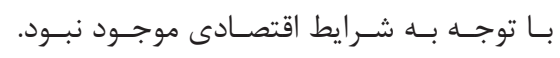

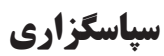

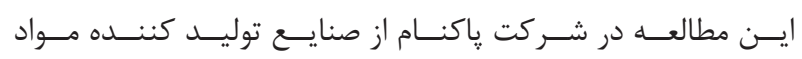

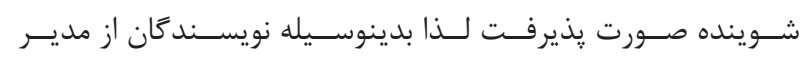

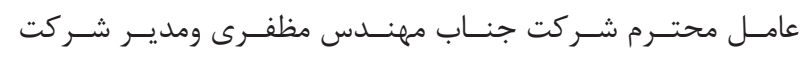

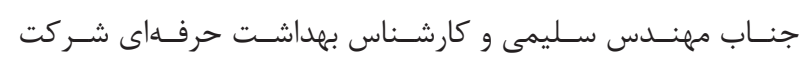

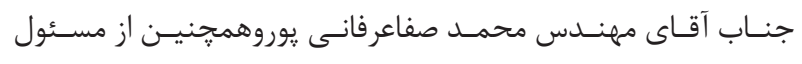

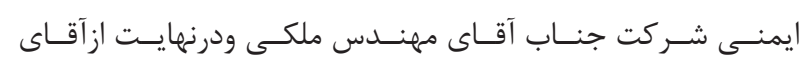

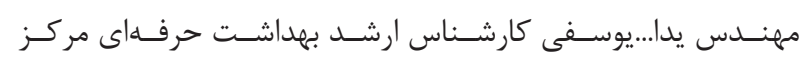

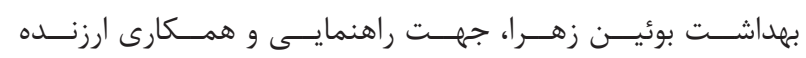

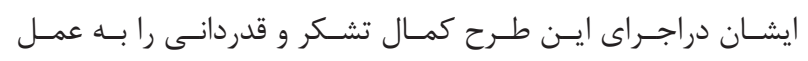
مى آورنــد.
اختـلالات اسـكلتى عضلانسى در بيـن كاركنـان شـود [• [ب]. نتايسج

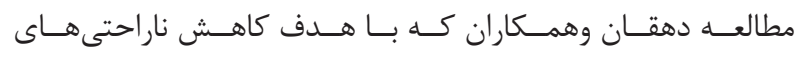

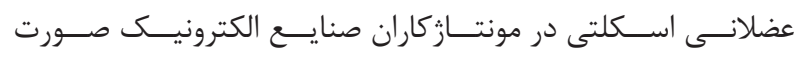

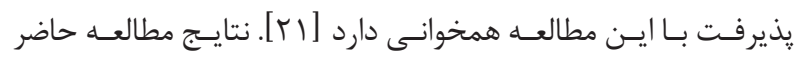

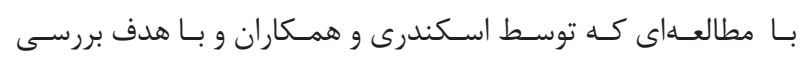

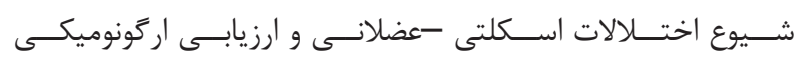

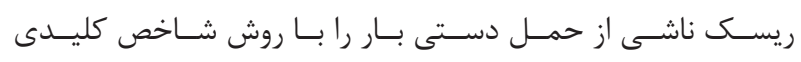
KIM

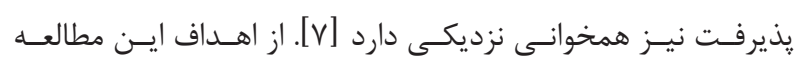

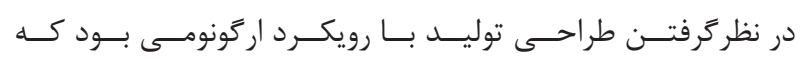

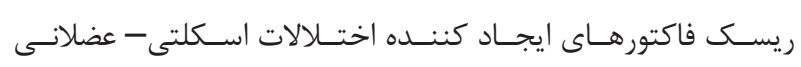

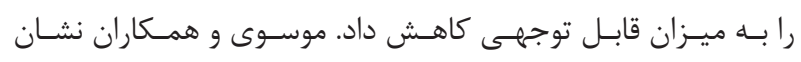

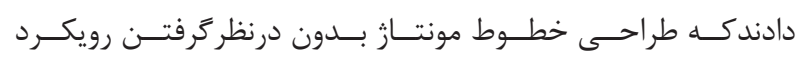

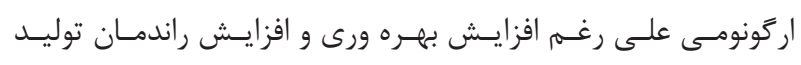

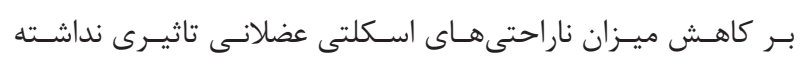

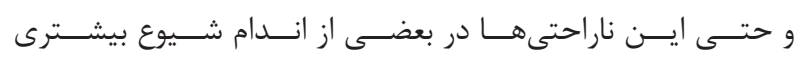

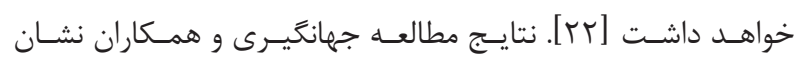

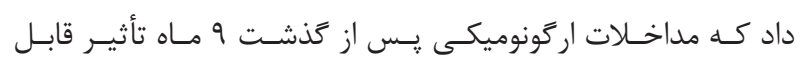

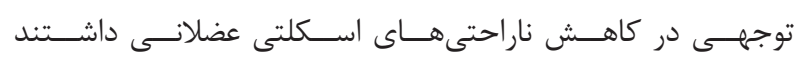

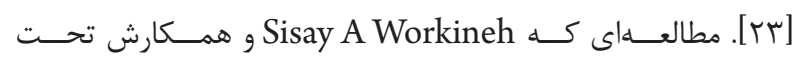

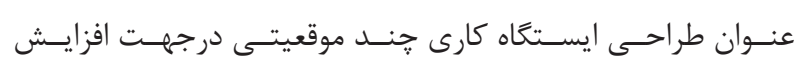

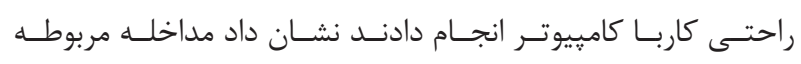

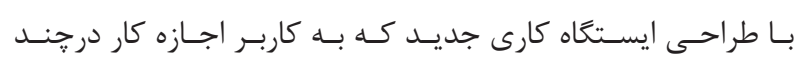

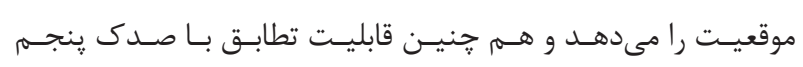

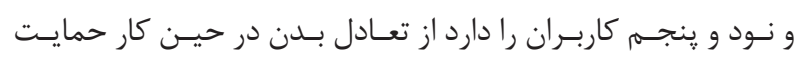

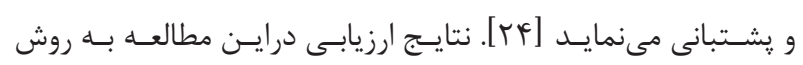

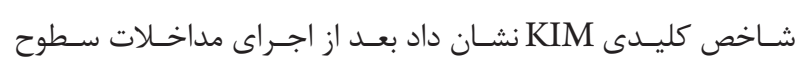

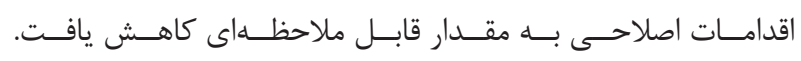

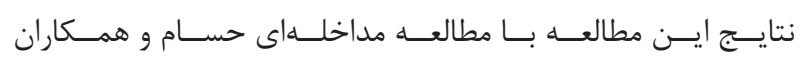

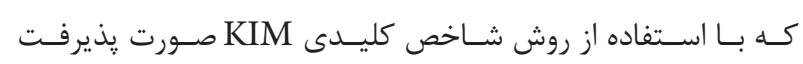

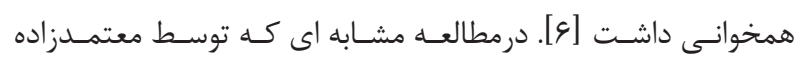

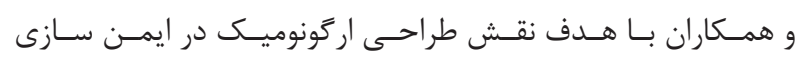

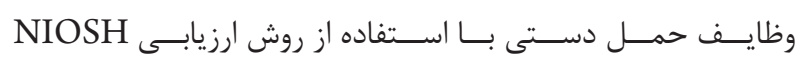

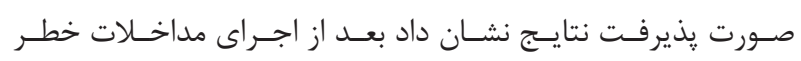

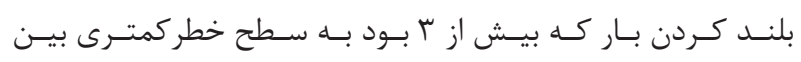

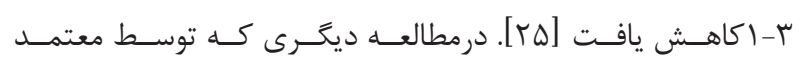

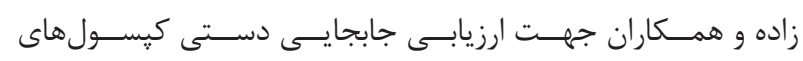

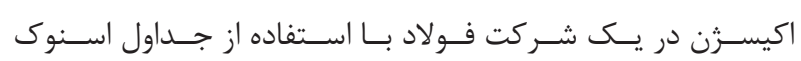

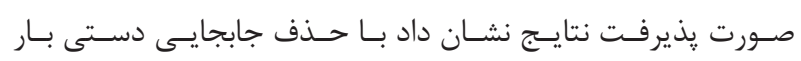

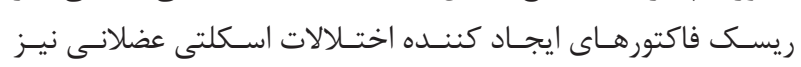




\section{REFERENCES}

1. Mohseni Bandpi M, Ahmadshirvani M, Bagheri M, Khalilian A. [A pathological investigation of back pain in nurse]. J Babol Med Uni. 2004;26(2):6.

2. Mattila M, Vilkki M. The Occupational Ergonomics Handbook: OWAS methods; 1999. 447-59 p.

3. Choobineh A, Lahmy M, Shahnvaz H. Musculoskeletal disorders - muscular carpet industry in Iran. J Public Health Instit Health Res. 2005;2:1-2.

4. Tajvar AH, Madani A, Farahnak M, Ghanbarnejhad A. Prevalence of Musculoskeletal and Cumulative Trauma Disorders in Aluminum Industry. $J$ Prev Med. 2014; 1(1):39-45.

5. Brisson C, Montreuil S, Punnett $L$. Effects of an ergonomic training program on workers with video display units. Scand J Work Environ Health. 1999;25(3):255-63. PMID: 10450777

6. Hesam G, Motamedzade M, khakbaz G, Moradpour Z. Ergonomics intervention in poultry slaughter industry and evaluate the effectiveness by key indicators method (KIM). J Ergon. 2014;2(2):9-19.

7. Falaki H, Motallebi Kashani M, Bahrami A. The prevalence of musculoskeletal disorders and occupational risk factors in Kashan SAIPA automobile industry workers by key indicator method (KIM), 1390. J Health Saf Work. 2012;2(1):27-36.

8. Kumar Kushwaha D, Kane PV. Ergonomic assessment and workstation design of shipping crane cabin in steel industry. Int J Ind Ergon. 2016;52:29-39.

9. Loo HS, Yeow PH. Effects of two ergonomic improvements in brazing coils of air-handler units. Appl Ergon. 2015;51:383-91. DOI: 10.1016/j.apergo.2015.06.007 PMID: 26154237

10. Amick BC, 3rd, Robertson MM, DeRango K, Bazzani L, Moore A, Rooney T, et al. Effect of office ergonomics intervention on reducing musculoskeletal symptoms. Spine (Phila Pa 1976). 2003;28(24):2706-11. DOI: 10.1097/01. BRS.0000099740.87791.F7 PMID: 14673374

11. Albers J, Estill C, MacDonald L. Identification of ergonomics interventions used to reduce musculoskeletal loading for building installation tasks. Appl Ergon. 2005;36(4):42739. DOI: 10.1016/j.apergo.2004.07.005 PMID: 15892937

12. Afifehzadeh-Kashani H, Choobineh A, Bakand SH, Gohari MR, Abbastabar H, Moshtaghi P. Validity and Reliability Farsi Version Cornell Musculoskeletal Discomfort Questionnaire (CMDQ). Instit Hosp. 2011;7(4):10-0.

13. Hejazi E, Sarmad Z, Bazargan A. Research ways of behavioral sciences. Tehran: Agah Publications; 2014.

14. Keikha Moghaddam AA. Ergonomics Assessment Methods. 2012.

15. Klussmann A, Steinberg U, Liebers F, Gebhardt H, Rieger MA. The Key Indicator Method for Manual Handling Operations (KIM-MHO) - evaluation of a new method for the assessment of working conditions within a cross-sectional study. BMC Musculoskelet Disord. 2010;11:272. DOI: 10.1186/1471-2474-11-272 PMID: 21108790

16. Marras WS, Karwowski W. Fundamentals and assessment tools for occupational ergonomics. Second Edition ed. USA: CRC Press; 2006.

17. Bridger RS. Introduction to Ergonomic. USA: Taylor \& Francis 2003.

18. Kalkis H, Roja Z, Kalkis V. Physical load analysis in hotel cleaning work. Agron Res. 2014;12(3):843-50.

19. Steinberg U. New tools in Germany: development and appliance of the first two KIM ("lifting, holding and carrying" and "pulling and pushing") and practical use of these methods. Work. 2012;41 Suppl 1(Supplement 1):3990-6. DOI: 10.3233/WOR-2012-0698-3990 PMID: 22317333

20. Mesbah F, Choobineh A, Tozihian T, Jafari P, Naghib-alhosseini F, Shidmosavi M, et al. Ergonomic intervention effect in reducing musculoskeletal disorders in staff of Shiraz Medical School. Instit Hosp. 2012;9(1):41-51.

21. Dehghan $\mathbf{N}$, Choobineh AR, Hasanzadeh J. Interventional ergonomic study to correct and improve working postures and decrease discomfort in assembly workers of an electronic industry. Iran Occup Health J. 2013;9(4):71-9.

22. Musavi F, Saremi M, Alibabaei A. The effect of assembly line redesign based on engineering techniques on productivity and ergonomics factors. Instit Hosp. 2016;12(6):1-15.

23. Jahangiri M, Mohammadpour H, Mosavi S, Saeidi CH, Negahban SAR, FarrajiTomarkandi V, et al. Concurrent Ergonomics Intervention and Implementation of Engineering and Administrative Techniques to Reduce Musculoskeletal Disorders in a Lead Mine. Arums Health. 2013;4(2):13446.

24. Workineh SA, Yamaura H. Effects of Multiple Working Positions on User Comfort: A Study on Multi-position Ergonomic Computer Workstation. Proc Manuf. 2015;3:4792-9. DOI: $10.1016 /$ j.promfg.2015.07.585

25. Motamedzade M, Dormohammadi A, Amjad Sardrodi H, Zarei E, Dormohammadi R, Shafii Motlagh M. The Role of Ergonomic Design and Application of NIOSH Method in Improving the Safety of Load Lifting Tasks. Arak Med Univ J. 2013;16(6):90-100.

26. Motamedzadeh M, Shafiei Motlagh M, Darvishi E. Ergonomics intervention in manual handling of oxygen. $J$ Health Saf Work. 2013;3(1):19-28.

27. Chanchai W, Songkham W, Ketsomporn P, Sappakitchanchai P, Siriwong W, Robson MG. The Impact of an Ergonomics Intervention on Psychosocial Factors and Musculoskeletal Symptoms among Thai Hospital Orderlies. Int J Environ Res Public Health. 2016;13(5). DOI: 10.3390/ ijerph13050464 PMID: 27153076 


\title{
Physical Ergonomic Assessment by Key Indicator Index (KIM) and Ergonomics Intervention in a Detergent-Producing Industry
}

\author{
Majid Motamedzade ${ }^{1}$, Asghar Payoon ${ }^{2, *}$, Rashid Heydari \\ Moghaddam ${ }^{3}$, Javad Fradmal ${ }^{4}$, Mohammad Babamiri ${ }^{3}$, Payam \\ Heydari $^{5}$
}

${ }^{1}$ Professor, Department of Ergonomics, School of Health, Hamedan University of Medical Sciences, Hamedan, Iran

${ }^{2} \mathrm{MSc}$, School of Health, Hamedan University of Medical Sciences, Hamedan, Iran

${ }^{3}$ Research Center for Health Sciences and Department of Ergonomics, School of Public Health, Hamadan University of Medical Sciences, Hamadan, Iran

${ }^{4}$ Department of Biostatistics and Modeling of Noncommunicable Diseases Research Center, School of Public Health, Hamedan University of Medical Sciences, Hamedan, Iran

${ }^{5} \mathrm{MSc}$, Department of Occupational Heealth Engineering, School of Health, Qazvin University of Medical Sciences, Qazvin, Iran

* Corresponding author: Asghar Payoon, MSc, Department of Ergonomics, School of

DOI: $10.21859 /$ joe- 05016 Health, Hamedan University of Medical Sciences, Hamedan, Iran.E-mail: a.payoon@ yahoo.com

Received: 30.01.2017

Accepted: 08.05.2017

\section{Keywords:}

KIM

Ergonomic Intervention Musculoskeletal Disorders Cornell Questionnaire

\section{How to Cite this Article:} Motamedzade M, Payoon A, Heydari Moghaddam R, Fradmal J, Babamiri M, Heydari P. Physical Ergonomic Assessment by Key Indicator Index (KIM) and Ergonomics Intervention in a Detergent-Producing Industry. $J$ Ergo 2016;5(1):43-50. DOI: $10.21859 /$ joe-05016

(c) 2017 Hamedan University of Medical Sciences.

\section{Abstract}

Introduction: Muscle-skeletal disorders are one of the most common job-related disorders in industrial workplaces due to different reasons. The current study performed an ergonomic assessment using the key indicator index (KIM) in order to reduce injuries in the bottle-making salon of one of the detergent-producing industries of Qazvin province. Methods: This study was an interventional study and consisted of 25 workers and was done in a bottle-making salon of one of the detergent-producing industries, during year 2016. For data collection, the Cornell questionnaire and key indicator index assessment method were used before and after the ergonomic interventions. Data was analyzed by descriptive analytical tests using the SPSS 16 software.

Results: According to the results, in the bottle production task (risk score: 4), the risk score decreased significantly by redesign (risk score: 2). Also, regarding the bottle's material, supply task that workers used to do it by manual material handling (risk score: 3 ), by redesign, risk score decreased significantly (risk score: 1 ).

Conclusions: In this study, musculoskeletal disorders decreased meaningfully by workstation ergonomic redesign after 5 months from the intervention. 